\title{
Un estudio de caso en el humedal de Agua Amarilla, Chile
}

\section{A case study in the Agua Amarilla wetland, Chile}

\author{
Eduardo Antonio Jaime Muñoz \\ Universidad Nacional de San Juan, Argentina. \\ ejaime18@gmail.com | 0000-0003-4412-0664
}

Para citar este artículo: Muñoz, E. (2021). Un estudio de caso en el humedal de Agua Amarilla, Chile. Entorno Geográfico, (22), 144-161. https://doi.org/10.25100/eg.v0i22.11403

\begin{abstract}
Resumen
Se presenta un estudio relacionado con la flora y fauna de los humedales precordilleranos, situados en la comuna de Monte Patria, Región de Coquimbo, Chile, específicamente en el sector de Agua Amarilla, Pedregal; sitios que presentan cambios debido a las transformaciones en su ambiente. El objetivo del trabajo es conocer e identificar la flora y fauna nativa que crece en los humedales y las actividades humanas que están influyendo en su degradación. La metodología de trabajo utilizada es trabajo de campo, uso de variados materiales de apoyo. Los resultados del trabajo son el hallazgo de 21 especies habitan el lecho y orillas del humedal, 27 especies vegetales en los alrededores del humedal y 29 especies de aves, las encuestas muestran una preocupación de los habitantes por el deterioro y la conservación.
\end{abstract}

Palabras clave: Territorio, humedales, actividades económicas, biodiversidad y comunidades rurales.

\begin{abstract}
A study related to the flora and fauna of the foothills of the Andes is presented, located in the Monte Patria commune, Coquimbo Region, Chile, specifically in the Agua Amarilla sector, Pedregal; sites that present changes due to transformations in their environment. The objective of the work is to know and identify the native flora and fauna that grow in wetlands and the human activities that are influencing their degradation. The work methodology used is field work, use of various support materials. The results of the work are the finding of 21 species inhabiting the bed and edges of the wetland, 27 plant species around the wetland and 29 species of birds, the surveys show a concern of the inhabitants for deterioration and conservation.
\end{abstract}

Keywords: Territory, wetlands, economic activities, biodiversity and rural communities.

Recibido: 17 de diciembre de 2020

Aceptado: 08 de abril de 2021

\section{Introducción}

Los humedales son un tipo de ecosistema difícil de definir debido a la existencia de una gran variedad de ellos, a su carácter altamente dinámico, a la dificultad de definir con precisión sus límites, a su localización y a la influencia humana (Ministerio de Medio Ambiente, 2010). En lugares montañosos los humedales altoandinos y ribereños, son importantes sitios para la conservación de la biodiversidad, estos espacios territoriales son reservorios de agua acumulada en forma de nieve en la alta cordillera. Los humedales son ecosistemas que entregan beneficios 
y servicios fundamentales para toda la vida sobre la tierra. Proveen agua dulce, alimentos y recursos; controlan las crecidas, representan la recarga de aguas subterráneas y son el hogar permanente o de paso, de muchas especies de flora y fauna (Ministerio de Medio Ambiente, 2018).

En este sentido los humedales entregan una diversidad de beneficios a los ecosistemas naturales, permitiendo la sobrevivencia de una variedad de organismos que viven en estos espacios naturales. Debido a esta razón el trabajo toma importancia, ya que en la zona de estudio hay un desconocimiento de las especies nativas de flora y fauna que existen en este lugar, ante esta problemática se plantea elaborar el siguiente manuscrito con el fin de conocer y registrar los vegetales y animales silvestres que se encuentran en la zona.

Se desarrolla un trabajo de campo donde se realizaron caminatas a lo largo de todo el humedal, observando las plantas y los animales que estaba presentes en la unidad de estudio, también se aplicaron 15 entrevistas semiestructuradas a personas de la comunidad del sector con el fin de conocer algunas impresiones de las personas, que viven en los alrededores del humedal.

En estos humedales alto andinos y ribereños, la degradación del hábitat natural, producto de las actividades humanas con la deforestación de plantas nativas para la plantación de monocultivos, la construcción de caminos para la minería sobre áreas naturales y la formación de microbasurales han ido degradando las áreas naturales, amenazando la flora y la fauna existe en el humedal Agua Amarilla. Por esta razón se ha planteado la siguiente pregunta de investigación. ¿Cómo es la composición florística y faunística del humedal y que factores inciden en su degradación?

\section{Estado de la cuestión}

La diversidad ecológica existente en humedales altoandinos es un factor relevante para la toma de decisiones que tengan como fin la protección y el cuidado de estos ecosistemas naturales amenazados por las actividades humanas que se dan en zonas rurales. Un humedal ribereño se conoce por la existencia de un bosque esclerófilo (Jorquera, et al, 2012), esta definición puede ser útil para poder distinguir un humedal con estas características. Unos de los problemas ambientales presentes en este tipo de humedales es la presencia de microbasurales siendo unos de los impactos y amenazas que se ve con mayor frecuencia en los humedales (Corporación de Ambientes Acuáticos de Chile, 2005). Este problema ha ido invadiendo los espacios naturales y alterando los ecosistemas naturales de las zonas ribereñas.

Si bien, el espacio geográfico de Agua Amarilla, es un caso donde se puede observar cómo la industria agrícola también ha ido reduciendo la superficie de los humedales, provocando una problemática ambiental, esto se puede articular con la publicación de Inversiones extraterritoriales, crisis ambiental y acción colectiva de América Latina (Ospina, et al, 2015)" donde se menciona las estrategias utilizadas por las empresas extractivistas, por apropiarse de los recursos naturales, como ha sido la creación de nuevas instituciones que brindaron a los actores extraterritoriales un acceso seguro a recursos naturales clave".

Este planteamiento aplicado a la realidad que viven muchas localidades rurales en Chile, puede ser visto al observar la llegada de adelantos que permiten mejorar la calidad de vida de las comunidades rurales en estudio. Sin embargo, la implementación de estos proyectos de inversión en el territorio, ha originado por un lado consecuencias negativas para el medio ambiente, esto puede ser visto en el humedal Agua Amarilla a través de la aparición de las empresas de telefonía, que han instalado las antenas celulares en los alrededores del humedal, 
promoviendo la construcción de caminos sobre áreas naturales lo que ha alterado la vida silvestre en el lugar.

Por mucho tiempo los humedales localizados en zonas rurales fueron considerados como lugares poco productivos y no tomados en cuenta e incluso como zonas riesgosas e insalubres a lo que era mejor drenar para convertir en área productivas tales como campos de cultivo, represas, terrenos urbanos u otros tipos de uso tradicional (CONAF, 2010). En esta lógica pareciera ser que los humedales han sido espacios poco valorados por las personas situación que ha producido una falta de preocupación por establecer medidas que contribuyan a su conservación.

Con el tiempo las consecuencias de las actividades desarrolladas por el hombre en los humedales, han provocado, una disminución de las aguas, baja cobertura vegetal que permite el desarrollo de los ciclos naturales de los seres vivos y escasa productividad en los cultivos agrícolas que dependen de su existencia, estos antecedentes permiten afirmar que los humedales cumplen un valor ecológico y económico para diversas comunidades en la tierra.

Para (Hardin, 2005), en su obra La tragedia de los comunes, menciona que los recursos naturales se rigen bajo la lógica general de abundancia y existencia ilimitada", Sin duda este pensamiento que ha sido instaurado por décadas en las zonas rurales, lo que producido un escaso interés de las comunidades por cuidar los recursos naturales.

Por otro lado, autores como (Ostrom, 1990) evidencian que dentro de ciertos grupos sociales es posible que exista la cooperación y responsabilidad colectiva sobre la explotación de los recursos naturales", Si bien el planteamiento de Ostrom, pareciera ser una oportunidad para las personas que viven alrededor del humedal Agua Amarilla, se requiere un trabajo continuo, donde la comunidad se debe comprometer a cuidar este espacio natural.

Para Jaime Cuevas Investigador en Planificación y Ordenamiento Territorial del Centro Científico de (CEAZA, 2020) recalca la importancia de la vegetación que crece en la zona de los humedales ya que filtra los nutrientes y contaminantes que provienen de actividades industriales, asentamientos humanos, agricultura y actividad forestal.

En este sentido el humedal de Agua Amarilla se caracteriza por poseer una estructura dada principalmente por los componentes que los constituyen, como la flora y fauna nativa que los rodea y los componentes abióticos y bióticos que se generan en cada uno de ellos (SAG, 2006). Un elemento muy notorio en la estructura de este tipo de humedales es la flora donde se puede distinguir una diversidad de plantas como nativas y endémicas.

Considerando este aspecto es interesante notar la importancia de la vegetación nativa en los humedales y su rol ecológico para el desarrollo de diversas aves, mamíferos, reptiles y anfibios que viven en estas y como estas plantas no solo son importantes para permitir la sobrevivencia de la fauna silvestre, sino también como elementos naturales que ayudan a descontaminar los humedales en estas zonas rurales.

En este aspecto el presente artículo propone un estudio en el humedal de Agua Amarilla ubicado en la comuna de Monte Patria con el fin de conocer la flora y fauna que existe en el humedal y que factores están incidiendo en la degradación de estos humedales.

\subsection{Descripción del área de estudio}

Los humedales existentes en la zona de Agua Amarilla son parte del rio Mostazal y se caracterizan por estar localizados en una zona donde el curso de agua, baja de la alta cordillera hacia los sectores bajos de la cuenca en forma de un rio que permite por un lado la sobrevivencia 
de una diversidad de organismos y por otra parte el desarrollo de actividades agrícolas donde trabaja la población rural de la zona.

En la zona del humedal de Agua Amarilla es muy común la plantación agrícola de uva de mesa. Según el Informe Odepa (Oficina de Estudios y Políticas Agrarias del Ministerio de Agricultura, del Gobierno de Chile (2018), la información censal de los frutales registra que el $20 \%$ de la superficie regional está dedicada al sector silvoagropecuario siendo ocupada con frutales pudiendo observar que siete especies frutales; uva de mesa, palto, clementina, olivo, nogal, limonero, naranjo son las de mayor relevancia en cuanto a superficie, tanto a nivel regional como en la relación región/país. Las comunas donde se localiza más del 65\% de la superficie de este grupo son: Ovalle y Monte Patria, en la provincia de Limarí, y Vicuña, en la provincia de Elqui.

Desde el punto de vista biogeográfico el humedal Agua Amarilla también favorece el desarrollo de una biodiversidad que depende de la existencia de este espacio geográfico. "En este sentido un humedal se origina donde el agua se halla en la superficie terrestre o donde la tierra está cubierta por aguas poco profundas (Jorquera, et al, 2012).

En la (Figura 1) se puede observar el humedal en el rio Mostazal y en sus alrededores el sector de Agua Amarrilla de Pedregal, comuna de Monte Patria, provincia del Limarí, Región de Coquimbo, Chile.

Fuente: Elaboración propia, 2020.

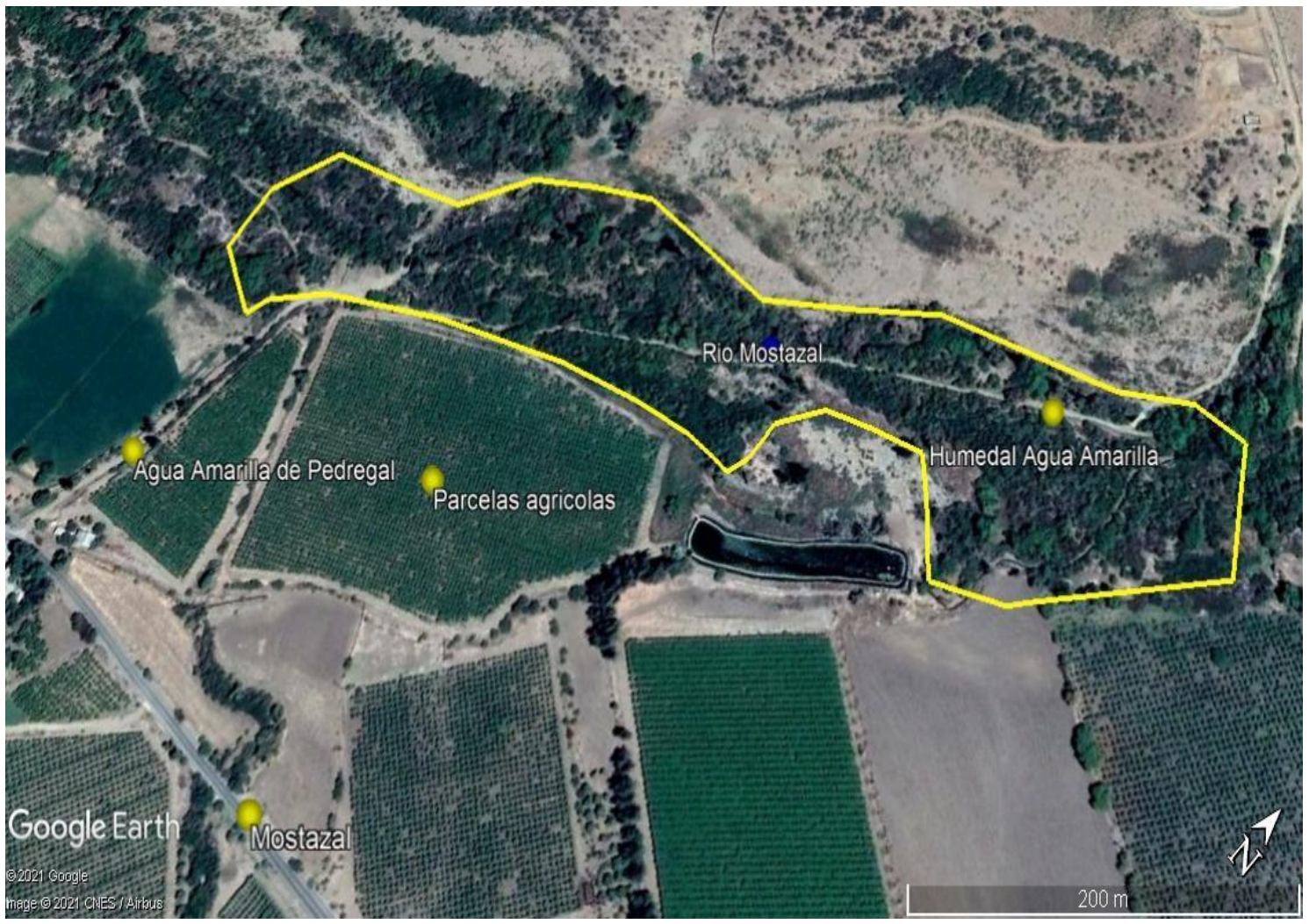

Figura 1. Mapa del Humedal Agua Amarilla

Como se observa en el mapa el humedal de Agua Amarilla pertenece a la media montaña del norte chico, en este lugar la alta cordillera acaba bruscamente hacia el Oeste en las proximidades de $\operatorname{los} 70^{\circ} 45^{\circ} \mathrm{W}$, a partir de este el relieve empieza a descender hacia los sectores bajos de la cuenca hidrográfica (Paskoff, 1993). 
Una geografía escabrosa y con presencia de altas cumbres son las formas características, que identifican al valle de Mostazal, donde es posible observar la presencia de una vegetación densa en la parte baja del valle y en la parte alta la existencia de un paisaje árido, donde es posible diferenciar formaciones vegetales espinosas debido a la escasa disponibilidad de agua que existe en esta parte del relieve geográfico.

\section{Métodos y materiales}

Los métodos utilizados para la identificación de las plantas son la ayuda de guías de campo de flora y fauna. Se realizó un análisis bibliográfico donde se usan libros de flora y fauna nativa como: Conociendo la flora nativa que crece alrededor de mi escuela (Jaime et al, 2020), Trepadoras y parásitas en el cerro La Olla, un estudio etnobotánico en la comuna de Monte Patria, Región de Coquimbo, Chile" (Jaime \& Villaseñor, 2016), Flora nativa de valor ornamental, identificación y propagación. Zona Central (Riedermann \& Aldunate, 2001) y Cactáceas en la flora silvestre de Chile (Hoffman, 2004).

Para la identificación de la fauna silvestre se utilizaron libros como Aves de Chile, un libro también para niños (Hoffman, et al, 2000), Aves de Chile (Jaramillo, 2015), La importancia de los humedales del rio Mostazal, comuna de Monte Patria-Región de Coquimbo”. (Jorquera, et al, 2012), Fauna Andina. Historia natural y conservación (Bonacic \& Ibarra, 2010). El puma en el altiplano de Tarapacá. Fauna Austral (Bonacic, et al, 2015).

Con la ayuda del programa computacional Microsoft Word, 2016, se confeccionó una entrevista semiestructurada y se aplican a 15 entrevistas a personas de la comunidad en los alrededores del humedal Agua Amarilla, se entrevistó a personas adultas y ellas en forma voluntaria decidieron participar del estudio. El contacto con ellas fue a través de visitas domiciliarias donde a través de conversaciones, contaron sus impresiones en relación al humedal Agua Amarilla.

Se revisó bibliografía especializada en metodología de investigación en geografía como Metodología de Cualitativa (Ruiz, 2012) y Metodología de la investigación en geografía (Bozzano et al, 2016). Se realizaron tres caminatas para identificar la flora y la fauna existente en el humedal Agua Amarilla, el horario fue de 9 de la mañana a 12 del día, la idea era avistar la mayor cantidad de organismos, que salen a posar sobre los árboles a esa hora. En estas caminatas participaron 2 personas en estación de verano, la actividad se repitió en dos oportunidades y la información fue registrada en tablas, utilizando el programa Microsoft Word, 2016.

\section{Resultados}

4.1. A continuación, en la (Tabla 1). Se expone el listado de especies encontradas en el lecho y orillas de del humedal presentes en el sector de sector de Agua Amarilla de Pedregal.

Tabla 1. Plantas nativas, introducidas y endémicas en el humedal Agua Amarilla.

\begin{tabular}{|l|l|l|l|l|l|l|l|}
\hline $\begin{array}{l}\text { Nombre } \\
\text { común }\end{array}$ & Nombre científico & Hierba & Arbusto & Árbol & Nativo & Introducido & Endémico \\
\hline Sauce llorón & $\begin{array}{l}\text { Salix babylonica } \\
\text { L. }\end{array}$ & & & $\mathrm{x}$ & & $\mathrm{x}$ & \\
\hline $\begin{array}{l}\text { Sauce } \\
\text { chileno }\end{array}$ & $\begin{array}{l}\text { Salix } \\
\text { Willd. }\end{array}$ & & $\mathrm{x}$ & $\mathrm{x}$ & & \\
\hline
\end{tabular}




\begin{tabular}{|c|c|c|c|c|c|c|c|}
\hline $\begin{array}{l}\text { Nombre } \\
\text { común }\end{array}$ & Nombre científico & Hierba & Arbusto & Árbol & Nativo & Introducido & Endémico \\
\hline Maitén & $\begin{array}{l}\text { Maytenus boaria } \\
\text { Molina }\end{array}$ & & & $\mathrm{x}$ & $\mathrm{X}$ & & \\
\hline Chilca & $\begin{array}{l}\text { Baccharis } \\
\text { calliprinos } \\
\text { Griseb }\end{array}$ & & $\mathrm{x}$ & & & & $\mathrm{x}$ \\
\hline Culén & $\begin{array}{l}\text { Otholobium } \\
\text { glandulosum (L.) } \\
\text { J.W. Grimes }\end{array}$ & & $\mathrm{x}$ & & $\mathrm{x}$ & & \\
\hline Molle & $\begin{array}{l}\text { Schinus } \\
\text { polygamus (Cav.) } \\
\text { Cabrera }\end{array}$ & & $\mathrm{x}$ & & $\mathrm{x}$ & & \\
\hline Palqui & $\begin{array}{l}\text { Cestrum parqui } \\
\text { L'Hér. }\end{array}$ & & $\mathrm{x}$ & & $\mathrm{x}$ & & \\
\hline Chacay & $\begin{array}{l}\text { Discaria trinervis } \\
\text { (Gillies ex Hook. } \\
\text { \& Arn.) Reiche }\end{array}$ & & $\mathrm{x}$ & & $\mathrm{x}$ & & \\
\hline Nipa & $\begin{array}{l}\text { Escallonia } \\
\text { illinita } \text { C. Presl } \\
\text { var. illinita }\end{array}$ & & $\mathrm{x}$ & & & & $\mathrm{x}$ \\
\hline Arrayán & $\begin{array}{l}\text { Luma chequen } \\
\text { (Molina) A. Gray }\end{array}$ & & $\mathrm{x}$ & & & & $\mathrm{x}$ \\
\hline Berro & $\begin{array}{l}\text { Nasturtium } \\
\text { officinale } \mathrm{R} . \mathrm{Br} .\end{array}$ & $\mathrm{x}$ & & & & & \\
\hline Hierba buena & $\begin{array}{l}\text { Mentha spicata } \\
\text { L. }\end{array}$ & $\mathrm{x}$ & & & & & \\
\hline Paico & $\begin{array}{l}\text { Dysphania } \\
\text { ambrosioides (L.) } \\
\text { Mosyakin \& } \\
\text { Clemants }\end{array}$ & $\mathrm{x}$ & & & & & \\
\hline Carrizal & $\begin{array}{l}\text { Phragmites } \\
\text { australis (Cav.) } \\
\text { Trin. ex Steud. }\end{array}$ & $\mathrm{x}$ & & & $\mathrm{x}$ & & \\
\hline Ortiga & $\begin{array}{l}\text { Loasa placei } \\
\text { Lindl. }\end{array}$ & $\mathrm{x}$ & & & $\mathrm{x}$ & & \\
\hline Espino & $\begin{array}{l}\text { Acacia caven } \\
\text { (Molina) Molina }\end{array}$ & & $\mathrm{x}$ & & $\mathrm{x}$ & & \\
\hline Cortadera & $\begin{array}{l}\text { Ceratophyllum } \\
\text { demersumem }\end{array}$ & & $\mathrm{x}$ & & $\mathrm{x}$ & & \\
\hline Álamo & Populus alba $L$ & & & $\mathrm{x}$ & $\mathrm{x}$ & $\mathrm{x}$ & \\
\hline Alfalfa & $\begin{array}{l}\text { Medicago sativa } \\
\mathrm{L}\end{array}$ & $\mathrm{x}$ & & & & $\mathrm{x}$ & \\
\hline
\end{tabular}




\begin{tabular}{|l|l|l|l|l|l|l|l|}
\hline $\begin{array}{l}\text { Nombre } \\
\text { común }\end{array}$ & Nombre científico & Hierba & Arbusto & Árbol & Nativo & Introducido & Endémico \\
\hline Cardo & $\begin{array}{l}\text { Silybum } \\
\text { marianum (L.) } \\
\text { Gaertn. }\end{array}$ & $\mathrm{x}$ & & & & $\mathrm{x}$ & \\
\hline Canutillo & $\begin{array}{l}\text { Equisetum } \\
\text { bogotense Kunth }\end{array}$ & $\mathrm{x}$ & & & & & \\
\hline
\end{tabular}

Fuente: Elaboración propia, 2020.

4.2. En la (Tabla 2). Se muestra el listado de especies encontradas alrededor del humedal de Agua Amarilla de Pedregal.

Tabla 2: Especies encontradas alrededor del humedal de Agua Amarilla de Pedregal.

\begin{tabular}{|c|c|c|c|c|c|c|c|}
\hline $\begin{array}{l}\text { Nombre } \\
\text { común }\end{array}$ & Nombre científico & Hierba & Arbusto & Árbol & Nativo & Introducido & Endémico \\
\hline Olivillo & $\begin{array}{l}\text { Spinoliva } \\
\text { ilicifolia (Hook. } \\
\text { \& Arn.) } \\
\text { G.Sancho subsp. }\end{array}$ & & & $\mathrm{x}$ & & & $\mathrm{x}$ \\
\hline Chañar & $\begin{array}{l}\text { Geoffroea } \\
\text { decorticans } \\
\text { (Gillies ex Hook. } \\
\text { \& Arn.) Burkart }\end{array}$ & & & $\mathrm{x}$ & & & $\mathrm{x}$ \\
\hline Espino & $\begin{array}{l}\text { Acacia caven } \\
\text { (Molina) Molina }\end{array}$ & & $\mathrm{x}$ & & $\mathrm{x}$ & & \\
\hline Quisco & $\begin{array}{l}\text { Echinopsis } \\
\text { chiloensis } \\
\text { (Colla) Friedrich } \\
\text { \& G.D. Rowley } \\
\text { subsp }\end{array}$ & & $\mathrm{x}$ & & & & $\mathrm{x}$ \\
\hline Pimiento & Schinus areira $\mathrm{L}$ & & & $\mathrm{x}$ & & $\mathrm{x}$ & \\
\hline Romero & $\begin{array}{l}\text { Baccharis } \\
\text { linearis (Ruiz \& } \\
\text { Pav.) Pers. subsp }\end{array}$ & & $\mathrm{x}$ & & $\mathrm{x}$ & & \\
\hline Chagual & $\begin{array}{l}\text { Puya alpestris } \\
\text { (Poepp.) Gay } \\
\text { subsp }\end{array}$ & & $\mathrm{x}$ & & & & $\mathrm{x}$ \\
\hline Varilla lisa & $\begin{array}{l}\text { Adesmia } \\
\text { argéntea Meyen }\end{array}$ & & $\mathrm{x}$ & & & & $\mathrm{X}$ \\
\hline Varilla brava & $\begin{array}{l}\text { Adesmia hystrix } \\
\text { Phil. }\end{array}$ & & $\mathrm{x}$ & & & & $\mathrm{x}$ \\
\hline
\end{tabular}




\begin{tabular}{|c|c|c|c|c|c|c|c|}
\hline $\begin{array}{l}\text { Nombre } \\
\text { común }\end{array}$ & Nombre científico & Hierba & Arbusto & Árbol & Nativo & Introducido & Endémico \\
\hline Mollaca & $\begin{array}{l}\text { Muehlenbeckia } \\
\text { hastulata (Sm.) } \\
\text { I.M. Johnst. var }\end{array}$ & & $\mathrm{x}$ & & & & $\mathrm{x}$ \\
\hline Algarrobo & $\begin{array}{l}\text { Prosopis } \\
\text { chilensis } \\
\text { (Molina) Stuntz } \\
\text { emend. Burkart }\end{array}$ & & & $\mathrm{x}$ & $\mathrm{x}$ & & \\
\hline Palqui & $\begin{array}{l}\text { Cestrum parqui } \\
\text { L’Hér }\end{array}$ & & $\mathrm{x}$ & & $\mathrm{x}$ & & \\
\hline Pucana & $\begin{array}{l}\text { Proustia } \\
\text { cuneifolia D. } \\
\text { Don subsp. } \\
\text { cinerea (Phil.) } \\
\text { Luebert }\end{array}$ & & $\mathrm{x}$ & & & & $\mathrm{x}$ \\
\hline Guayacán & $\begin{array}{l}\text { Porlieria } \\
\text { chilensis I.M. } \\
\text { Johnst. }\end{array}$ & & $\mathrm{x}$ & & & & $\mathrm{x}$ \\
\hline Colliguay & $\begin{array}{l}\text { Colliguaja } \\
\text { odorífera Molina }\end{array}$ & & $\mathrm{x}$ & & & & $\mathrm{x}$ \\
\hline Abrojo & $\begin{array}{l}\text { Colletia hystrix } \\
\text { Clos }\end{array}$ & & $\mathrm{x}$ & & & & $\mathrm{x}$ \\
\hline Sandillón & $\begin{array}{l}\text { Eriosyce aurata } \\
\text { (Pfeiff.) Backeb. } \\
\text { var. }\end{array}$ & & $\mathrm{x}$ & & & & $\mathrm{x}$ \\
\hline Chapin & $\begin{array}{l}\text { Maihueniopsis } \\
\text { ovata (Pfeiff.) F. } \\
\text { Ritter }\end{array}$ & & $\mathrm{x}$ & & & & $\mathrm{x}$ \\
\hline Paltos & $\begin{array}{l}\text { Persea } \\
\text { americana Mill }\end{array}$ & & & $\mathrm{x}$ & & $\mathrm{x}$ & \\
\hline Damasco & $\begin{array}{l}\text { Prunus } \\
\text { armeniaca } L\end{array}$ & & & $\mathrm{x}$ & & $\mathrm{x}$ & \\
\hline Durazno & Prunus pérsica $L$ & & & $\mathrm{x}$ & & $\mathrm{x}$ & \\
\hline Nogales & Juglans regia $L$ & & & $\mathrm{x}$ & & $\mathrm{x}$ & \\
\hline Parras & $\begin{array}{l}\text { Vitis vinífera } \\
\text { sativa }\end{array}$ & & & $\mathrm{x}$ & & $\mathrm{x}$ & \\
\hline
\end{tabular}




\begin{tabular}{|l|l|l|l|l|l|l|l|}
\hline $\begin{array}{l}\text { Nombre } \\
\text { común }\end{array}$ & Nombre científico & Hierba & Arbusto & Árbol & Nativo & Introducido & Endémico \\
\hline Alfalfa & $\begin{array}{l}\text { Medicago sativa } \\
\text { L }\end{array}$ & $\mathrm{x}$ & & & & $\mathrm{x}$ & \\
\hline Álamos & Populus alba L & & & $\mathrm{x}$ & & $\mathrm{x}$ & \\
\hline Paico & $\begin{array}{l}\text { Dysphania } \\
\text { ambrosioides s } \\
\text { (L.) Mosyakin \& } \\
\text { Clemants }\end{array}$ & $\mathrm{x}$ & & & & $\mathrm{x}$ & \\
\hline Chépica & $\begin{array}{l}\text { Agrostis } \\
\text { capillaris }\end{array}$ & $\mathrm{x}$ & & & $\mathrm{x}$ & \\
\hline
\end{tabular}

Fuente: Elaboración propia, 2020.

4.3. En la (Tabla 3). Listado de la fauna silvestre avistada en las orillas del humedal Agua Amarilla de Pedregal, se incorporan especies confirmadas es decir aquellas especies que fueron avistadas en las caminatas y especies esperadas según el libro La importancia de los humedales del río Mostazal, comuna de Monte Patria-Región de Coquimbo (Jorquera, et al, 2012).

Tabla 3. Fauna silvestre avistada en las orillas del humedal Agua Amarilla de Pedregal,

\begin{tabular}{|c|c|c|c|c|c|c|c|}
\hline $\begin{array}{l}\text { Nombre de } \\
\text { aves y } \\
\text { mamíferos } \\
\text { silvestres }\end{array}$ & $\begin{array}{l}\text { Nombre } \\
\text { científico }\end{array}$ & $\begin{array}{l}\text { Aves } \\
\text { comunes }\end{array}$ & $\begin{array}{l}\text { Aves } \\
\text { rapac } \\
\text { es }\end{array}$ & $\begin{array}{l}\text { Mamíferos } \\
\text { carnívoros }\end{array}$ & Roedor & $\begin{array}{l}\text { Especies } \\
\text { confirmadas }\end{array}$ & $\begin{array}{l}\text { Especies } \\
\text { Esperadas }\end{array}$ \\
\hline Turca & $\begin{array}{l}\text { Pteroptochos } \\
\text { megapodius }\end{array}$ & $\mathrm{x}$ & & & & $\mathrm{x}$ & \\
\hline Tucúquere & $\begin{array}{l}\text { Bubo } \\
\text { magellanicus }\end{array}$ & & $\mathrm{x}$ & & & & $\mathrm{x}$ \\
\hline Carpinterito & $\begin{array}{l}\text { Veniliornis } \\
\text { lignarius }\end{array}$ & $\mathrm{x}$ & & & & $\mathrm{x}$ & \\
\hline Diuca & Diuca diuca & $\mathrm{x}$ & & & & $\mathrm{x}$ & \\
\hline Tordo & $\begin{array}{l}\text { Curaeus } \\
\text { curaeus }\end{array}$ & $\mathrm{x}$ & & & & $\mathrm{x}$ & \\
\hline Chuncho & $\begin{array}{l}\text { Glaucidium } \\
\text { nanum }\end{array}$ & & $\mathrm{x}$ & & & & $\mathrm{x}$ \\
\hline Perdiz & $\begin{array}{l}\text { Nothoprocta } \\
\text { perdicaria }\end{array}$ & $\mathrm{x}$ & & & & & $\mathrm{x}$ \\
\hline Zorzal & $\begin{array}{l}\text { Turdus } \\
\text { falcklandii } \\
\text { magellanicus }\end{array}$ & $\mathrm{x}$ & & & & $\mathrm{x}$ & \\
\hline Diucón & $\begin{array}{l}\text { Xolmis pyrope } \\
\text { pyrope }\end{array}$ & $\mathrm{x}$ & & & & $\mathrm{x}$ & \\
\hline
\end{tabular}




\begin{tabular}{|c|c|c|c|c|c|c|c|}
\hline $\begin{array}{l}\text { Nombre de } \\
\text { aves y } \\
\text { mamíferos } \\
\text { silvestres }\end{array}$ & $\begin{array}{l}\text { Nombre } \\
\text { científico }\end{array}$ & $\begin{array}{l}\text { Aves } \\
\text { comunes }\end{array}$ & $\begin{array}{l}\text { Aves } \\
\text { rapac } \\
\text { es }\end{array}$ & $\begin{array}{l}\text { Mamíferos } \\
\text { carnívoros }\end{array}$ & Roedor & $\begin{array}{l}\text { Especies } \\
\text { confirmadas }\end{array}$ & $\begin{array}{l}\text { Especies } \\
\text { Esperadas }\end{array}$ \\
\hline Fio-Fio & $\begin{array}{l}\text { Elaenia } \\
\text { albiceps } \\
\text { chilensis }\end{array}$ & $\mathrm{x}$ & & & & $\mathrm{x}$ & \\
\hline Peuco & $\begin{array}{l}\text { Parabuteo } \\
\text { unicinctus } \\
\text { unicinctus }\end{array}$ & & $\mathrm{x}$ & & & $\mathrm{x}$ & \\
\hline Loica & $\begin{array}{l}\text { Sturnella } \\
\text { loyca }\end{array}$ & $\mathrm{x}$ & & & & $\mathrm{x}$ & \\
\hline Tórtola & $\begin{array}{l}\text { Zenaida } \\
\text { auriculata } \\
\text { auriculata }\end{array}$ & $\mathrm{x}$ & & & & $\mathrm{x}$ & \\
\hline Huairavo & $\begin{array}{l}\text { Nycticorax } \\
\text { nycticorax } \\
\text { obscurus }\end{array}$ & $\mathrm{x}$ & & & & & $\mathrm{x}$ \\
\hline Piden & $\begin{array}{l}\text { Pardirallus } \\
\text { sanguinolentus } \\
\text { landbecki }\end{array}$ & & & & & $\mathrm{x}$ & \\
\hline $\begin{array}{l}\text { Garza } \\
\text { grande }\end{array}$ & Ardea alba & $\mathrm{x}$ & & & & $\mathrm{x}$ & \\
\hline Águila & $\begin{array}{l}\text { Geranoaetus } \\
\text { melanoleucus } \\
\text { australis }\end{array}$ & & $\mathrm{x}$ & & & & $\mathrm{x}$ \\
\hline Chercán & $\begin{array}{l}\text { Troglodytes } \\
\text { aedon } \\
\text { chilensis }\end{array}$ & & & & & $\mathrm{x}$ & \\
\hline Garza chica & Egretta thula & $\mathrm{x}$ & & & & & $\mathrm{X}$ \\
\hline Tiuque & $\begin{array}{l}\text { Milvago } \\
\text { chimango } \\
\text { chimango }\end{array}$ & & $\mathrm{x}$ & & & & $\mathrm{X}$ \\
\hline Chingolito & $\begin{array}{l}\text { Zonotrichia } \\
\text { capensis } \\
\text { chilensis }\end{array}$ & $\mathrm{x}$ & & & & $\mathrm{x}$ & \\
\hline $\begin{array}{l}\text { Loro } \\
\text { Tricahue }\end{array}$ & $\begin{array}{l}\text { Cyanoliseus } \\
\text { patagonus } \\
\text { bloxami }\end{array}$ & $\mathrm{x}$ & & & & $\mathrm{x}$ & \\
\hline Cóndor & Vultur gryphus & & $\mathrm{x}$ & & & & $\mathrm{X}$ \\
\hline $\begin{array}{l}\text { Pato corta } \\
\text { corrientes }\end{array}$ & $\begin{array}{l}\text { Merganetta } \\
\text { armata armata }\end{array}$ & $\mathrm{x}$ & & & & & $\mathrm{x}$ \\
\hline
\end{tabular}




\begin{tabular}{|c|c|c|c|c|c|c|c|}
\hline $\begin{array}{l}\text { Nombre de } \\
\text { aves y } \\
\text { mamíferos } \\
\text { silvestres }\end{array}$ & $\begin{array}{l}\text { Nombre } \\
\text { científico }\end{array}$ & $\begin{array}{l}\text { Aves } \\
\text { comunes }\end{array}$ & $\begin{array}{l}\text { Aves } \\
\text { rapac } \\
\text { es }\end{array}$ & $\begin{array}{l}\text { Mamíferos } \\
\text { carnívoros }\end{array}$ & Roedor & $\begin{array}{l}\text { Especies } \\
\text { confirmadas }\end{array}$ & $\begin{array}{l}\text { Especies } \\
\text { Esperadas }\end{array}$ \\
\hline Codorniz & $\begin{array}{l}\text { Callipepla } \\
\text { californica } \\
\text { brunnescens }\end{array}$ & $\mathrm{x}$ & & & & $\mathrm{x}$ & \\
\hline Pequén & $\begin{array}{l}\text { Athene } \\
\text { cunicularia } \\
\text { cunicularia }\end{array}$ & & $\mathrm{x}$ & & & & $\mathrm{x}$ \\
\hline Golondrina & $\begin{array}{l}\text { Tachycineta } \\
\text { meyeni }\end{array}$ & $\mathrm{x}$ & & & & & $\mathrm{x}$ \\
\hline Tagua & $\begin{array}{l}\text { Fulica } \\
\text { armillata }\end{array}$ & $\mathrm{x}$ & & & & & $\mathrm{x}$ \\
\hline Lechuza & Tyto alba & & $\mathrm{x}$ & & & & $\mathrm{x}$ \\
\hline León & $\begin{array}{l}\text { Puma } \\
\text { concolor puma }\end{array}$ & & & $\mathrm{x}$ & & & $\mathrm{x}$ \\
\hline $\begin{array}{l}\text { Gato } \\
\text { andino }\end{array}$ & $\begin{array}{l}\text { Leopardus } \\
\text { jacobita }\end{array}$ & & & $\mathrm{x}$ & & & $\mathrm{x}$ \\
\hline Zorro chilla & $\begin{array}{l}\text { Lycalopex } \\
\text { griseus }\end{array}$ & & & $\mathrm{x}$ & & $\mathrm{x}$ & \\
\hline $\begin{array}{l}\text { Zorro } \\
\text { culpeo }\end{array}$ & $\begin{array}{l}\text { Lycalopex } \\
\text { culpaeus }\end{array}$ & & & $\mathrm{x}$ & & & $\mathrm{x}$ \\
\hline $\begin{array}{l}\text { Ratón cola } \\
\text { de pincel }\end{array}$ & Octodon degus & & & & $\mathrm{x}$ & & $\mathrm{x}$ \\
\hline
\end{tabular}

Fuente: Elaboración propia, 2020.

Diseño y aplicación de las entrevistas

La confección de las entrevistas se realizó tomando en considerando las observaciones realizadas durante las salidas a terreno en el humedal Agua Amarilla, se visualizó las problemáticas ambientales que presenta este espacio natural debido a las actividades humanas que están afectando a este lugar natural, tomando en consideración estas observaciones se plantearon preguntas a los entrevistados.

El diseño de la entrevista se realizó utilizando el programa computacional Microsoft Word 2016, en un documento se escribieron las preguntas de las entrevistas y luego se imprimieron para poder aplicarlas.

\section{Recolección de datos}

Se realizaron visitas a domiciliarias a los pobladores que tienen sus casas en las cercanías del humedal Agua Amarilla, se conversa con ellos, se toma el tiempo necesario para poder escuchar sus relatos y experiencias de vida, en un ambiente de mucho respeto y entendimiento con las personas, ellos dieron el consentimiento que sus respuestas fueran consideradas en el trabajo. Luego de registrar la información en las entrevistas, se trabaja con el programa Microsoft Excel (2016), en la confección de las (Figuras 4, 5, 6 y 7) que se presentan a continuación. 
Fuente: Elaboración propia, 2020.

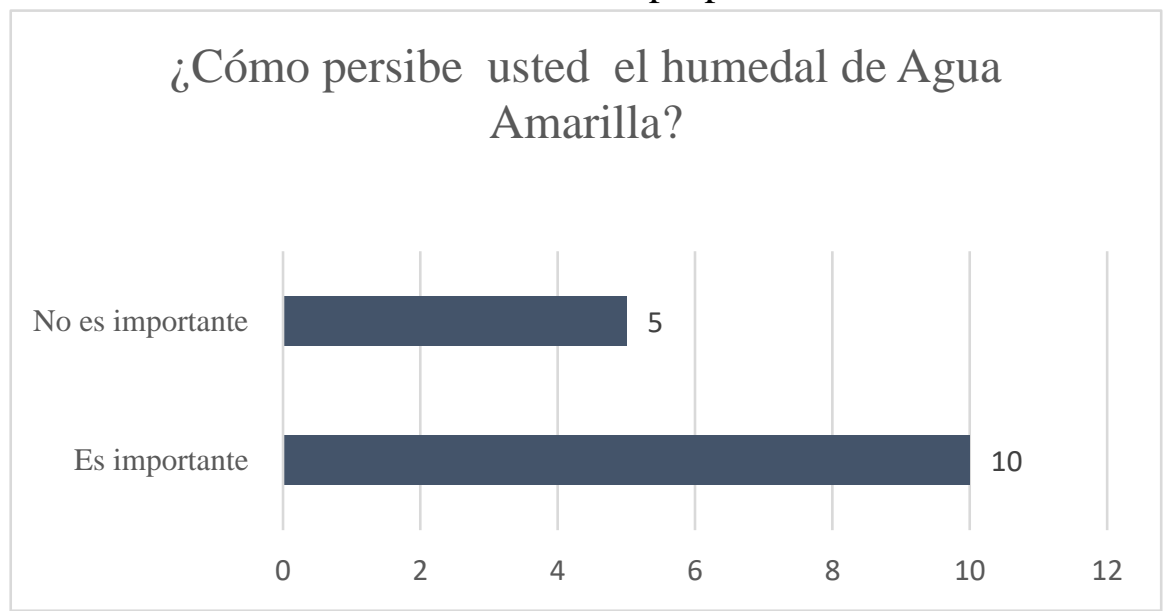

Figura 4. Percepción de los entrevistados en relación al humedal Agua Amarilla.

Fuente: Elaboración propia, 2020

¿Qué le gusta más del humedal de Agua Amarilla

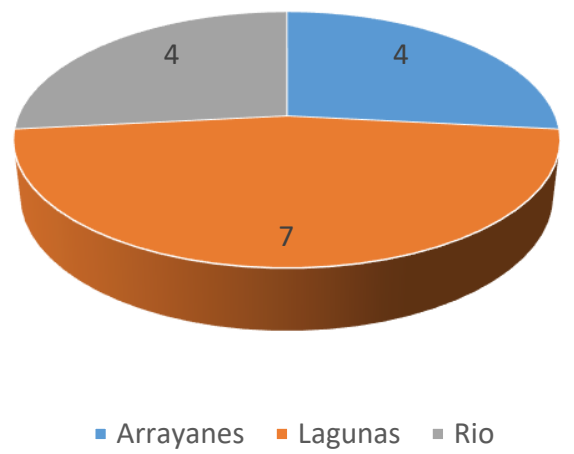

Figura 5. Elementos que más llaman la atención de las personas

Fuente: Elaboración propia, 2020.

¿Qué problemas ambientales cree usted que estan presentes en el humedal de Agua Amarilla?

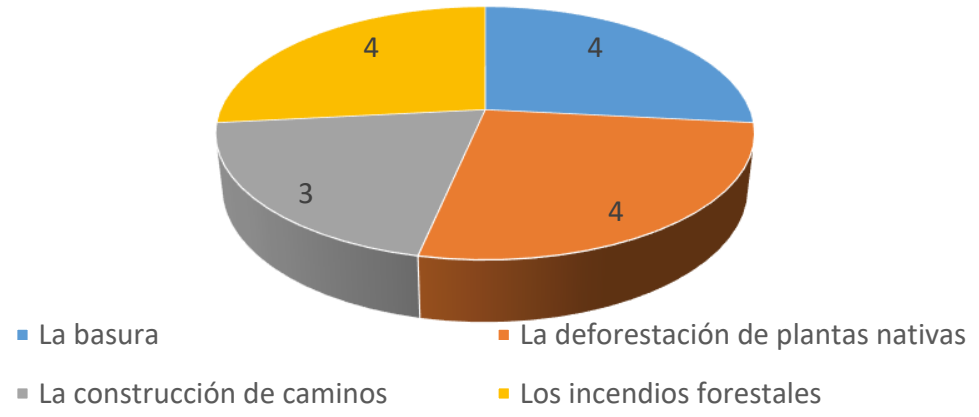

Figura 6. Problemas ambientales que están presentes en el humedal de Agua Amarilla, información obtenida a partir de los datos entregados por la comunidad. 
Fuente: Elaboración propia, 2020.

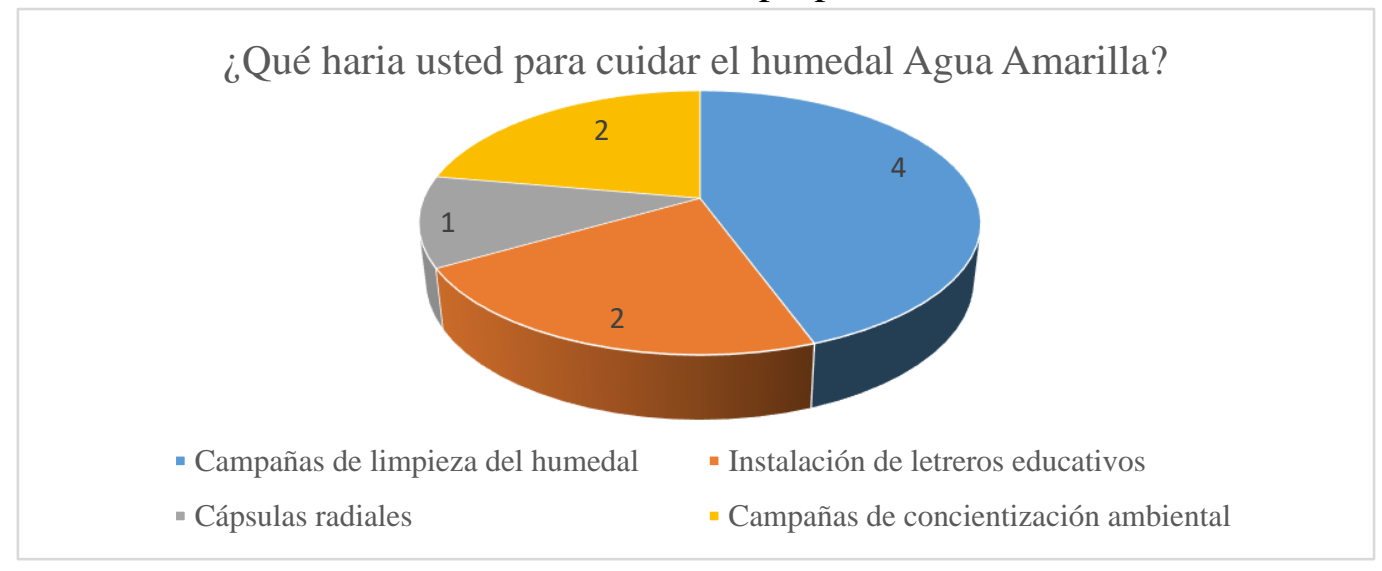

Figura 7. Iniciativas comunitarias para cuidar y proteger el humedal Agua Amarilla

\section{Discusión y resultados finales}

\subsection{Plantas en el lecho y orillas del humedal Agua Amarilla}

En el humedal presente en el sector de Agua Amarilla de Pedregal, se encontraron en el lecho y en la orilla de los mismos un total de 21 de plantas, entre ellas 8 son hierbas, 9 son arbustos, 4 son árboles, siendo nativas 11 de ellas, introducidas 5 y 3 endémicas. Estos vegetales proveen a diversos seres vivos de alimento, refugio y protección frente a los depredadores.

Respecto a la cantidad de arbustos encontrados en el lecho y orillas del humedal en el listado arbustos destaca el paico por sus propiedades. "La planta tiene un uso medicinal y es utilizada para aliviar dolores intestinales (Hoffman et al, 1992). En este sentido la presencia de plantas como el caso del paico es un factor recurrente en los humedales que existen en la zona, los lugareños utilizan la planta para preparar infusiones con el fin de aliviar el dolor de estómago. Las semillas del paico son esparcidas por las cabras y animales de carga que comen los brotes tiernos de la planta y llevan los granos hacia otras zonas, donde estos vegetales empiezan a crecer, siendo recurrente observar plantas de paicos en los potreros cercanos a Agua Amarilla, donde los cabreros pastan sus cabras.

Un árbol introducido que se puede apreciar en el paisaje del humedal Agua Amarilla es el sauce llorón "esta planta fue introducida en Europa a fines del siglo XVII, y desde ahí en América. Hoy en Chile está ampliamente distribuido (Servicio Nacional del Patrimonio, 2019). El sauce llorón se encuentra distribuido alrededor de todo el humedal Agua Amarilla, a pesar que es una especie introducida, ha conseguido colonizar las orillas del lecho del río, donde es posible encontrar árboles frondosos con troncos gruesos y un follaje que permite a los turistas y personas de la comunidad poder tomar sombra y disfrutar de la tranquilidad del lugar con sus familiares. El palqui es un arbusto nativo que crece en suelos degradados y secos, en los bordes de caminos (Hoffmann et al, 1992), a pesar que el vegetal se desarrolla en suelos poco fértiles y degradados por la acción humana, la planta presenta un uso medicinal los lugareños de la zona de Agua Amarilla buscan sus ramas para utilizar en la limpieza de los hornos de pan y baños para aliviar dolores reumáticos. La chilca es una planta muy común en la zona de los humedales, el arbusto crece al borde del lecho del río Mostazal, debido a la estructura leñosa, muchas aves construyen sus nidos en las ramas del vegetal. Según (Jaime \& Villaseñor, 2016), los lugareños de zonas rurales utilizan la leña seca del vegetal para hacer encender los hornos de barro donde se hace pan amasado y las abejas de miel se alimentan del néctar y polen de la flor del vegetal. 


\subsection{Plantas en los alrededores del humedal Agua Amarilla}

En esta área geográfica del humedal se encontraron 27 plantas entre las cuales se pueden distinguir 3 hierbas, 14 arbustos y 20 árboles, en relación a su categoría 4 de ellas son nativas, 9 son introducidas y 13 son endémicas. En comparación con las plantas encontradas en el lecho y el alrededor del humedal existe una mayor cantidad de plantas endémicas que las encontradas en el lecho y orillas del humedal.

En este sentido es importante destacar, cómo la presencia de las plantas introducidas en el lecho y orillas del humedal, están ligadas a la presencia de agua y humedal en el humedal Agua Amarilla. Un caso de esta situación es la que ocurre con los cardos: Silybum marianum (L.) Gaertn, esta especie introducida una vez que sus semillas son esparcidas en el suelo, empiezan a germinar y a cubrir espacios naturales que son el hábitat natural de especies nativas y endémicas.

Una especie endémica que crece de las laderas que se encuentran alrededor del humedal de Agua Amarilla, es el sandillón es una especie endémica de Chile desde la región de Atacama hasta la Metropolitana, en laderas de cerros y bordes de quebradas, a pleno sol, en suelos pedregosos (Riedermann \& Aldunate, 2001). Esta especie se ha adaptado a las condiciones de aridez presentes en el paisaje natural, la planta es una cactácea que guarda agua en sus tejidos cuando las escasas lluvias caen en la zona. Un árbol nativo presente en los alrededores del humedal es el algarrobo: Prosopis chilensis (Molina) Stuntz emend. Burkart este individuo suele crece sobre las planicies y laderas que se encuentran alrededor del humedal Agua Amarilla, cuando los cursos de agua empiezan a llegar al tronco de las plantas algunas malezas introducidas como la chépica empiezan a trepar sobre los troncos de estas vegetales. En las caminatas realizadas en los alrededores del humedal se observó en los potreros y predios agrícolas, la presencia de chépica: Agrostis capillaris la planta introducida que empieza atrapar algunas plantas nativas en la parte de sus troncos y raíces, este comportamiento biológico impide el desarrollo de los vegetales produciendo la muerte de las plantas débiles que no pueden desarrollarse y alcanzar sus ciclos naturales. (Quiroz, et al. 2009).

Sin duda la presencia de estas plantas introducidas han puesto en riesgo la sobrevivencia de las plantas nativas y endémicas que han crecido al largo de los años en estas zonas rurales como el área del humedal Agua Amarilla, una actividad que ha aumentado la presencia de crecimiento de estas plantas ha sido la crianza de cabras, ya que estos animales transportan las semillas de estas plantas, esparciendo a través de sus guanos, pepas de están vegetales sobre los terrenos de las parcelas agrícolas de la unidad de estudio.

\subsection{Fauna silvestre avistada en las orillas del humedal Agua Amarilla de Pedregal}

En el trabajo de campo realizado en el humedal Agua Amarilla, se encontró un total de 34 individuos de los cuales 29 son aves entre ellas 16 aves fueron confirmadas a través de los avistamientos en terreno realizado por los observadores y 18 se incorporaron al listado como especies esperadas. Dentro del grupo de las aves registradas 19 son aves comunes, 8 son aves rapaces.

El grupo de las aves avistadas en la zona del humedal destaca una bandada de tordos que volaba sobre la copa de los árboles los algarrobos, esta especie es un pájaro bullicioso que forma enormes bandadas en que todos parecen gritar al mismo tiempo (Hoffmann \& Lazo, 2000). 
Considerando este aspecto, los lugareños mencionan que este pájaro construye sus nidos de barro, paja y palos en las ramas de los algarrobos.

Los pidenes son aves que viven en todo tipo de humedales, como lagunas, lagos, ríos, esteros o canales de regadío, con vegetación donde protegerse (Martínez \& Gonzales 2016). Esta ave en la zona del humedal Agua Amarilla, fue vista buscando insectos entremedios de las plantas de berros, en los cauces de agua que nacen de las vertientes que alimentan al humedal, los pidenes en tiempo de reproducción colocan sus huevos, sobre las ramas de chilcas que bajan hacia el lecho del agua. Solo se registraron 4 mamíferos de los cuales 1 se confirmó su presencia en el humedal y 3 se incorporaron al listado con especies esperadas en este tipo de ecosistemas naturales. En relación con la fauna avistada destaca la presencia de mamíferos carnívoros como la zorra chilla y el zorro culpeo, estas especies dependen de la existencia de estos humedales para encontrar su alimento y poder alimentar a sus crías. "El zorro culpeo es un animal solitario y de hábitos crepusculares, aunque captura la mayor parte de sus presas en forma oportunista durante la noche (Iriarte \& Jaksica, 2012). Animales silvestres como el zorro culpeo utilizan el humedal Agua Amarilla para poder buscar alimento y defenderse de sus depredadores.

\subsection{Percepciones de las personas en torno al humedal de Agua Amarilla}

Se aplicó un total de 15 entrevistas semiestructuradas a lugareños que viven en los alrededores del humedal Agua Amarilla, en la primera pregunta de la entrevista semiestructurada se preguntó 1. ¿Cómo perciben el humedal Agua Amarilla? 5 personas consideraron que el humedal no es importante y 10 personas consideran que si, estos resultados permiten afirmar que dentro del grupo de los entrevistados existe una preocupación por el cuidado del humedal. La pregunta 2 ¿Qué le gusta más del humedal Agua Amarilla? 4 personas consideran los arrayanes, 5 lagunas y 4 el río, considerando estos datos, se puede decir que los entrevistados lo que más valoran del humedal son las lagunas que donde ellos suelen ir a bañarse junto a sus seres queridos y amigos en los veranos.

La pregunta 3 ¿Qué problemas ambientales cree usted que están presentes en el humedal de Agua Amarilla? 4 personas consideran que es la basura, 3 construcción de caminos, 4 deforestación de las plantas nativas y 4 incendios. En relación a esta pregunta la formación de microbasurales en algunos sectores del humedal de Agua Amarilla es una problemática ambiental presente en estos espacios rurales.

Según comentan las personas entrevistadas "la gente de otras localidades o ciudades viene a dejar basura sobre las plantas y a orillas del río eso provoca que el viento y los animales lleven estos residuos sobre las zonas naturales y algunos caen al cauce de agua produciendo la contaminación del río, donde muchas personas utilizan esta agua para darle a sus animales y regar sus sembrados.

Arroyave, et al (2006). Se refieren a los proyectos viales como lo es la construcción y mejoras de caminos han sido considerados como obras que representan un beneficio social y económico para las regiones y mejoran la calidad de vida de los habitantes.

Es interesante mencionar como los proyectos viales representan un beneficio para las comunidades rurales, llevando adelantos como un buen camino, una mejor conectividad o acceso a los servicios básicos como agua potable o electricidad. Sin embargo, el paso de un camino sobre zona donde existen humedales tiene un impacto negativo en la flora y fauna que depende de la existencia de estos lugares.

En este sentido los humedales desempeñan un papel fundamental desde una perspectiva ecológica y socioeconómica (Senhadji et al, 2017). Es importante mencionar como estos sitios 
son el hábitat natural de diversas aves y plantas que dependen de la existencia de estos lugares para poder sobrevivir. Considerando este aspecto es importante describir como la construcción de caminos en zonas donde existen humedales han ido modificando los hábitats naturales de distintas especies que dependen de condiciones naturales para su sobrevivencia. Un ejemplo de esta problemática ha sido la preocupación que existe de parte de algunos comuneros por la disminución de las áreas de crecimiento natural del canutillo, el vegetal es utilizado por las personas de la comunidad de Mostazal y sus alrededores para aliviar dolores a la vejiga y a los riñones, pero debido a la desaparición del bosque natural que lo cubre ha presentado una escasez siendo difícil encontrar nuevas plantas para uso medicinal.

Finalmente, en la pregunta 4 se les pregunta ¿Qué haría usted para cuidar el humedal Agua Amarilla? 4 personas consideran que se deberían promover campañas de limpieza del humedal, 1 cápsulas radiales, 2 instalación de letreros educativos y 4 campañas de concientización ambiental como charlas y talleres. Es importante considerar cómo las campañas de recolección de basura son unas de las actividades recurrentes para limpiar los humedales debido a la contaminación producida por las actividades humanas.

Considerando esta problemática el sitio virtual humedales Chiloé, menciona "buenas prácticas para cuidar y proteger los humedales entre ellas se destaca fomentar el reciclaje y reutilización de residuos domiciliarios, denunciar las descargar de contaminantes al medio ambiente.

Es importante mencionar cómo estas medidas contribuyen a mejorar la problemática ambiental, de los microbasurales presentes en zonas donde existe una diversidad vegetal y animal que aún se mantiene, pero debido a la amenaza de que tiene estas problemáticas para la vida silvestre del lugar hoy es necesario un cuidado y protección de estas áreas naturales.

\section{Referencias Bibliográficas}

Arroyave, M., Gómez, C., Gutiérrez, M., Múnera, D., Zapata, P., Vergara, I., Andrade, L., Ramos. K. (2006). Impacto de Las carreteras sobre la fauna silvestre y sus principales medidas de manejo. Revista EIA, (5), 45-57.

Bonacic, C., Leichtle, J., y Ohrens, O. (2015). El puma en el altiplano de Tarapacá. Fauna Austral. Facultad de Agronomía e Ingeniería Forestal, (pp. 1-74). Pontificia Universidad Católica de Chile.

Bonacic, C., y Ibarra, J. (2010). Fauna Andina. Historia natural y conservación. Laboratorio de Fauna Australis. Facultad de Agronomía e Ingeniería Forestal, (pp. 1-192). Pontificia Universidad Católica de Chile.

Bozzano, H., Frediani, J., Cirio, G., y Barrionuevo. C. (2016). Metodología de la Investigación en Geografía. Facultad de Humanidades y Ciencias de la Educación. Editorial de la Universidad de La Plata. Argentina.

Corporación Nacional Forestal. CONAF. (2010). Convenio de eficiencia institucional 2012. Programa Nacional para la Conservación de Humedales Insertos en el Sistema Nacional de Áreas Protegidas del Estado. Corporación Nacional Forestal. Gobierno de Chile.

Corporación de Ambientes Acuáticos de Chile. (2005). Manos al humedal. En Quevedo, L. (Ed) Guía práctica para escolares, (pp 52) Santiago de Chile.

Centro de Estudios Avanzados en Zonas Áridas. CEAZA (2020). Entrevista la vegetación de las riberas contribuye a descontaminación y contaminación del recurso hídrico por 
Jaime Cuevas Investigador en Planificación y Ordenamiento Territorial del Centro Científico. La Serena.

Hardin, G. (2005). La tragedia de los comunes. Polis Revista de la Universidad Bolivariana, 4(10), 11.

Hoffmann, A., y Lazo, I. (2000). Aves de Chile, un libro también para niños. Centro de Recursos Educativos Avanzados, CREA. Universidad Andrés Bello. Chile.

Hoffmann, A. (2004). Cactáceas en la flora silvestre de Chile. Ediciones Fundación Claudio Gay.

Hoffmann, A., Farga, C., Lastra, J., y Vechazi, E. (1992). Plantas medicinales de uso común en Chile, Tercera Edición. Ediciones Fundación Claudio Gay.

Jaramillo. A. (2015). Aves de Chile. Guía descubrir la naturaleza. Editorial Roberto Mandiola. Chile.

Jaime, E., \& R. Villaseñor (2016). Trepadoras y parasitas en el cerro La Olla, un estudio etnobotánico en la comuna de Monte Patria, Región de Coquimbo, Chile. Chloris Chilensis, (1).

Jaime. E., Villaseñor R., y Sandoval. A. (2020). Conociendo la flora nativa que crece alrededor de mi escuela. Proyecto Fondo de Protección Ambiental. Ministerio de Medio Ambiente, Chile.

Jorquera, A., C. Iturrieta, F. Sánchez, M. Valdés, M. Espinosa \& S. Espinosa. (2012). La importancia de los humedales del río Mostazal, comuna de Monte Patria. Junta vigilancia del río Mostazal y sus afluentes, Proyecto Fondo de Protección Ambiental FPA 4-G-O11-2012 El valle en nuestras manos: Junto protegiendo la biodiversidad de los humedales que mantienen nuestra vida y cultura. Chile.

Quiroz, C., Pauchard, A., Marticorena, A., Pauchard, A., y Cavieres, L. (2009). Manuel de Plantas Invasoras del Centro- Sur de Chile. Laboratorio de Invasiones Biológicas.

Martínez, D., y Gonzales, C. (2016). Las aves de Chile. Guía de Campo y breve historia natural. Ediciones del Naturalista.

Ministerio de Medio Ambiente (2010). La protección ambiental de los humedales costeros de la Región de Coquimbo. Fondo de Protección Ambiental del Ministerio de Medio Ambiente, Chile.

Ministerio de Medio Ambiente (2018). Plan Nacional de Protección de Los Humedales 20182022. Chile.pp33.

Riedemann, P., y Aldunate, G. (2001). Flora nativa de valor ornamental, identificación y propagación. Zona Central. Editorial Andrés Bello.

Ruiz. J (2012). Metodología de la investigación cuantitativa $5^{a}$ Edición. Universidad de Dousto.

Iriarte A \& Jaksica. F. (2012). Los carnívoros de Chile. Ediciones Flora y Fauna. Ediciones Flora \& Fauna-CASEB Pontificia Universidad Católica de Chile.

Oficina de Estudios y Políticas Agrarias. Odepa. (2018). Región de Coquimbo, información regional. https://bit.ly/3qmjpNv

Paskoff. R (1993). Geomorfología de Chile semiárido. Ediciones Universidad de La Serena.

Ospina. P. Bebbington. A. Hollenstein. P. Nussbaum. I \& Ramírez. E (2015). Inversiones extraterritoriales, crisis ambiental y acción colectiva en América Latina. Serie Documentos de Trabajo №166 1-27. Grupo de Trabajo Cohesión Territorial para el Desarrollo. Programa Cohesión Territorial para el Desarrollo. RIMISP. Centro Latinoamericano para el Desarrollo Rural. Santiago, Chile. 
Senhadji, K., RUÍZ, M., y Rodríguez, J. (2017). Estado ecológico de algunos humedales colombianos en los últimos 15 años: Una evaluación prospectiva. Colombia Forestal, 20(2), 181-191. http://dx.doi.org/10.14483/udistrital.jour.colomb.for.2017.2.a07

Ostrom. E (1990). El gobierno de los bienes comunes: la evolución de las instituciones de acción colectiva. Fondo de Cultura Económica. México.

Servicio Nacional del Patrimonio, (2019). Ficha técnica de sauce llorón en la página web del Servicio Nacional del Patrimonio, Chile. https://bit.ly/35RbpdK

Servicio Agrícola y Ganadero. SAG. (2006). Conceptos y criterios para la evaluación ambiental de humedales. Centro de Ecología Aplicada Ltda. 